\title{
Diarrhées intraitables dues à des anomalies congénitales des entérocytes
}

\author{
Alan D. Phillips \\ Centre de Gastro-entérologie pédiatrique, Royal Free Hospital, Londres, Royaume-Uni
}

\section{Mots-clés}

Diarrhée intraitable · Nourrisson · Entéropathie

\section{Résumé}

Contexte: Les diarrhées intraitables du nourrisson sont des affections sévères responsables d'une morbidité et d'une mortalité élevées. Ces cas demandent beaucoup de soins de la part des parents et des soignants, car il n'existe aucun traitement simple. Le petit nombre de ces patients laisse penser que seule une collaboration internationale regroupant les ressources et les sujets atteints, permettra de faire progresser la compréhension de ces affections. Des analyses régulières de la littérature dans ce domaine sont nécessaires pour faire connaître les progrès et discuter des hypothèses et critères diagnostiques. Méthodes: Le présent article est une analyse de la littérature concernant les anomalies congénitales des entérocytes. Résultats: Des diagnostics précis sont possibles dans ce groupe de maladies, et les critères en sont de plus en plus acceptés. Les cas publiés au plan mondial font apparaître des différences d'évolution clinique, certains de moindre sévérité. La morbidité et la mortalité demeurent cependant élevées. Conclusions: Un long chemin reste encore à parcourir afin de déterminer l'origine de ces troubles. La biologie moléculaire ouvre des perspectives prometteuses de détermination des anomalies génétiques de ces maladies. II s'agit d'une priorité, car il serait ainsi possible de disposer de critères diagnostiques plus précis, de préciser les relations de ces maladies entre elles et d'apporter les informations nécessaires sur leurs mécanismes.

Copyright @ 2006 Nestec Ltd., Vevey/S. Karger AG, Basel

\section{Introduction}

Les diarrhées intraitables demeurent des affections pédiatriques sévères, responsables d'une morbidité et d'une mortalité élevées. Elles représentent une charge majeure et demandent aux parents comme aux soignants de s'impliquer fortement dans les soins. La forme particulière de diarrhée intraitable abordée ici, due à des anomalies congénitales des entérocytes, affecte souvent des nourrissons au cours des premiers jours de leur vie. Les difficultés liées aux biopsies intestinales et aux abords veineux nécessaires au diagnostic et au traitement aggravent considérablement les problèmes que posent des pertes diarrhétiques quotidiennes qui peuvent excéder $10 \%$ du poids du corps. Un diagnostic précis est néanmoins possible. La nutrition parentérale a permis une survie relativement prolongée; la transplantation intestinale offre l'espoir d'une meilleure qualité de vie et les techniques de génétique moléculaire, celui de la découverte des anomalies génétiques qui sous-tendent certainement ces affections.

\section{KARGER}

Fax +4161306 1234 E-Mail karger@karger.ch www.karger.com
C 2006 Nestec Ltd. Vevey/S. Karger AG, Basel 0250-9644/06/0641-0015\$23.50/0

Accessible en ligne à www.karger.com/anf
Alan D. Phillips

Centre for Paediatric Gastroenterology

Lower 3rd Floor, Royal Free Hospital, Pond Street

Londres, NW3 2QG (UK)

Tel. +44 207830 2783, Fax +44 207830 2146, E-Mail a.phillips@medsch.ucl.ac.uk 

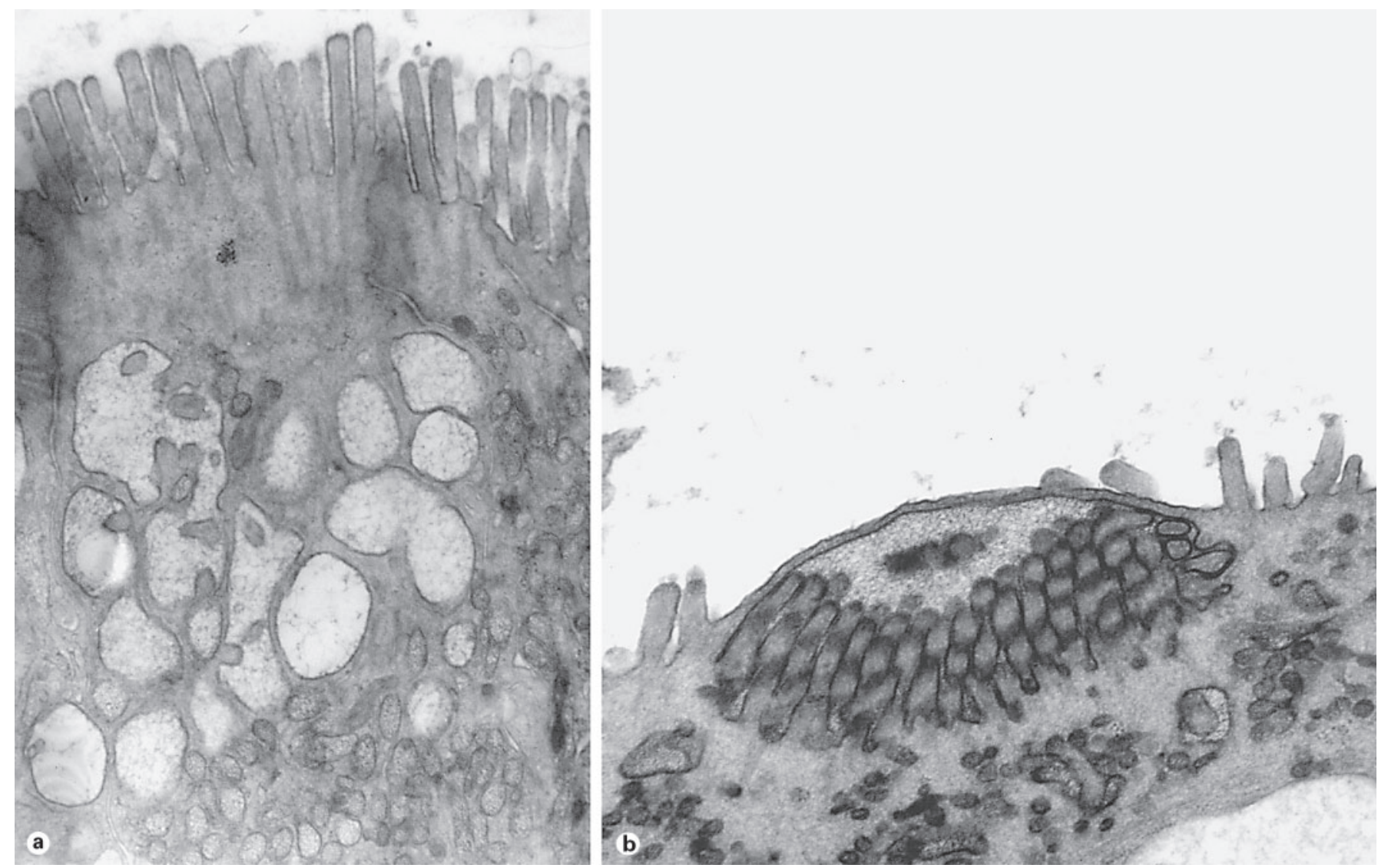

Fig. 1. Atrophie microvillositaire. a Développement apparent d'une microvillosité dans une cellule dont la bordure en brosse est intacte bien que courte. b Inclusion microvillositaire bulleuse; aspect d'inclusion.

Le terme de diarrhée intraitable a été introduit par Avery et coll. [1] au début des années 1960 à partir d'un groupe de 20 nourrissons âgés de moins de trois mois et présentant une diarrhée chronique (durée de plus de 14 jours) avec coproculture négative et persistant malgré des interventions médicales. Tous ont nécessité une nutrition parentérale et neuf sont décédés. Des cas similaires ont été publiés les années suivantes [2,3], les auteurs suggérant que l'affection était héréditaire et comportait des lésions intestinales, et que plusieurs entités pathologiques en étaient probablement responsables (entéropathie familiale [4] et diarrhée familiale prolongée fatale [5]).

Davidson et coll. [4] ont décrit l'entéropathie comme une atrophie villositaire avec hypoplasie des cryptes sans inflammation de la lamina propria, laissant entendre qu'une hyperplasie des cryptes (telle qu'observée dans la maladie coeliaque) et un mécanisme immunitaire étaient peu vraisemblables. Bien que des études ultérieures aient remis en question la nature hypoplasique de l'entéropa- thie (voir plus bas), cette observation a été généralisée et des critères immunohistochimiques ont été appliqués aux cas de diarrhée intraitable [6,7]. Cette approche a permis de distinguer les maladies à médiation immunitaire de celles liées à une anomalie épithéliale, ce qui a simplifié leur classification [7] et a permis d'optimiser les options thérapeutiques [8]. Le présent article est centré sur les affections liées à des anomalies épithéliales et n'abordera pas l'entéropathie auto-immune ni les troubles apparentés à médiation immunitaire ou leurs traitements, ces domaines étant traités ailleurs.

L'entérocolite ulcéreuse idiopathique du nourrisson $[9,10]$ n'est pas abordée non plus, car ses caractéristiques sont plus proches de celles d'une diarrhée intraitable d'origine immunitaire.

A ce jour, un petit nombre d'affections a été reconnu parmi les anomalies congénitales des entérocytes responsables d'une diarrhée intraitable. La qualité des données permettant de les placer dans cette catégorie est variable, 

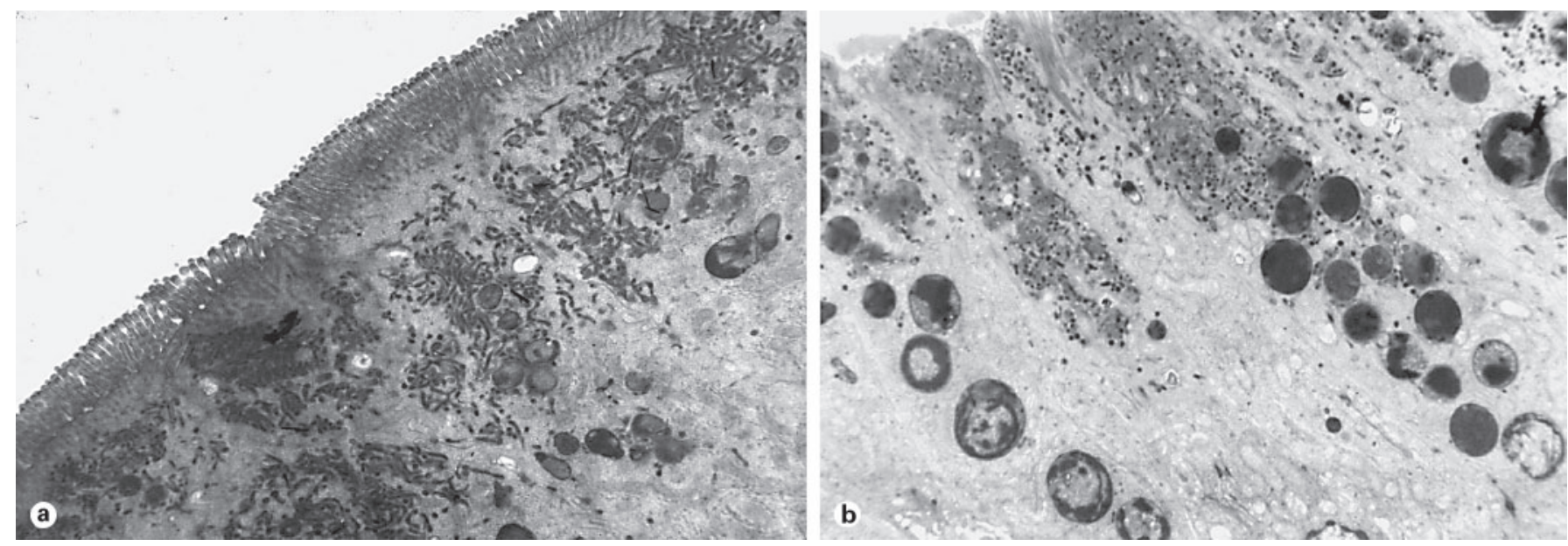

Fig. 2. Atrophie microvillositaire. Granules sécrétoires dans des cas à début tardif (a) ou atypique (b).

mais, au minimum, il existe une entéropathie avec peu ou pas d'inflammation dans la lamina propria. Les anomalies sous-jacentes n'ont pas été établies; des études sont en cours afin d'établir leurs bases génétiques moléculaires. Aucune anomalie génique n'a encore été découverte. Les principaux diagnostics que l'on peut évoquer sont l'atrophie microvillositaire ou maladie des inclusions microvillositaires, la dysplasie épithéliale intestinale («tufting enteropathy» des anglo-saxons), la diarrhée syndromique ou syndrome tricho-hépato-entérique, l'entérocolite ulcéreuse idiopathique du nourrisson et les troubles de la glycosylation, certains cas demeurant encore idiopathiques. Ils sont discutés plus bas. Plusieurs revues ont été publiées récemment concernant les diagnostics les plus fréquents dans le cadre des diarrhées intraitables du nourrisson [7, 9-15]; la présente analyse tentera d'éviter trop de redondances.

\section{Atrophie microvillositaire/maladie des inclusions microvillositaires}

Deux abstracts présentés en 1982 au congrès annuel de l'ESPGHAN ont rapporté des observations intrigantes effectuées sur l'épithélium de nourrissons atteints d'une diarrhée intraitable. Schmitz et coll. [16] ont proposé le terme d'atrophie microvillositaire, tandis que Goutet et coll. [17] se sont référés à Davidson et coll. [4] pour l'emploi du terme descriptif d'entéropathie familiale avec atrophie villositaire hypoplasique. Les deux équipes d'auteurs ont décrit des inclusions microvillositaires dans l'épithélium, comme l'avait fait Davidson et coll. [4] dans leur série de cinq nourrissons; elles ont de plus indiqué la présence de «granules sécrétoires» dans les cellules cryptiques et les entérocytes de surface [4]. Nous avons publié deux cas supplémentaires de diarrhée intraitable d'origine congénitale correspondant à une entéropathie présentant les mêmes aspects spécifiques (inclusions microvillositaires et augmentation des «granules sécrétoires»; fig. 1,2) et suggéré qu'il s'agissait de caractéristiques diagnostiques de la maladie [18]. Par la suite, Cutz et coll. [11] ont proposé le terme de maladies des inclusions microvillositaires afin de souligner l'anomalie des microvillosités.

\section{Signes cliniques}

Le tableau clinique est caractéristique, sévère et précoce, les troubles apparaissant généralement au cours de la première semaine de la vie $[4,11,12,18]$. La diarrhée persiste en l'absence de nutrition entérale et est exacerbée par l'alimentation orale [12]. La maladie est mondiale, des cas ayant été rapportés en Europe [12], au MoyenOrient [12], en Asie [19, 20] et en Amérique du Nord [11], y compris chez des Américains natifs (nation Navajo) [21]. Les descriptions provenant d'Afrique sont peu nombreuses, bien que l'auteur ait porté le diagnostic d'atrophie microvillositaire chez des descendants nord-américains d'Africains. Dans les pays en développement, la sévérité de la maladie peut ne pas permettre son diagnostic.

La majorité des cas survient au cours de la première semaine de la vie, mais les troubles apparaissent parfois 
après la période néonatale, et ces cas ont été qualifiés d'atrophie microvillositaire «à début tardif» [12]. Les anomalies morphologiques intestinales caractéristiques sont présentes, bien qu'avec de subtiles différences (voir plus bas), d'où le diagnostic. La survie à long terme est rare; on considère que le pronostic pourrait être meilleur dans les cas «à début tardif» [12]. Il est trop tôt pour admettre cette corrélation, bien que, dans une série de 23 patients, 3 des 5 survivants étaient des cas «à début tardif» [12]. Le patient $n^{\circ} 9$ de cette série est à présent âgé de 23 ans et son état est relativement satisfaisant sous nutrition parentérale partielle, mais sans amélioration des anomalies intestinales (Ventura A, communication personnelle).

La description de cas supplémentaires a permis de décrire des modes de début et des types d'évolution différents, intéressants, car pouvant permettre de progresser dans la compréhension des mécanismes sous-jacents et/ ou de rappeler la nécessité d'envisager le diagnostic dans des situations ne correspondant pas au tableau habituel, bien que la majorité des cas se présente de la façon décrite dans la littérature $[4,11,12,18]$. La plupart des cas ne sont pas associés à un polyhydramnios maternel, bien qu'un cas de polyhydramnios ait été récemment décrit en association à une occlusion intestinale prénatale qui s'est révélée être une atrophie microvillositaire [22]. Une atrophie microvillositaire doit donc être envisagée dans le cadre du diagnostic différentiel d'une diarrhée chlorée ou d'une anomalie des échanges $\mathrm{Na}-\mathrm{H}$ [24]. Une patiente atteinte d'une forme à «début tardif» (3 mois) avec les anomalies typiques a spontanément pris du poids à l'âge de 2 ans, malgré la persistance de la diarrhée, et la nutrition parentérale a pu être arrêtée à l'âge de 4 ans [25]. Cette évolution est inhabituelle et une transplantation intestinale précoce [26] n'aurait pas été justifiée chez elle ni dans d'autres cas «à début tardif».

Il est intéressant de rapporter qu'à la suite d'une biopsie hépatique, un patient en attente d'une transplantation hépatique et intestinale [27] a présenté une hémorragie intrapéritonéale sévère nécessitant une intervention chirurgicale; un choc hémorragique engageant le pronostic vital et une insuffisance rénale sont apparus. De façon remarquable la diarrhée a simultanément diminué et le patient n'a émis aucune selle pendant une semaine, puis le débit fécal a repris à $10-30 \mathrm{mg} / \mathrm{kg} /$ jour jusqu'à la transplantation. Une nutrition entérale a induit une augmentation du débit de selles sans modification de la morphologie intestinale. Une diminution extrême de la pression hydrostatique a apparemment modifié la nature sécrétoire de la diarrhée.
La maladie est transmise probablement comme un trait récessif autosomique en raison du nombre de parents consanguins et de la fréquente atteinte de frères et sœurs [12,28]. Des maladies génétiques simultanées ont été décrites [29, 30], mais il est difficile de savoir si elles sont la conséquence de l'atteinte des gènes contigus ou si elles sont héritées indépendamment. La fréquence de la consanguinité permet d'envisager des études de génétique moléculaire et la possibilité d'établir les bases génétiques de la maladie.

Des cas de diarrhée intraitable similaires sans aspect histologique d'atrophie microvillositaire pourraient représenter des cas apparentés, et suggèrent que les anomalies des microvillosités pourraient être variés [31, 32]. Elles ont été regroupées sous le terme de dystrophie microvillositaire intestinale [31]. Les aspects diagnostiques caractéristiques ont pu passer inaperçus [33]; le degré de similitude avec l'atrophie microvillositaire nécessite des études supplémentaires, dont des analyses de génétique moléculaire.

\section{Anatomopathologie}

Il existe une entéropathie avec atrophie villositaire, mais sans hyperplasie notable des cryptes, ce qui se traduit par un amincissement de la muqueuse [12]; il n'existe aucune inflammation de la lamina propria. Il ne s'agit pas d'une atrophie villositaire hyperplasique [4], car le nombre des cellules cryptiques est augmenté [12, 34], comme le sont l'apoptose et la prolifération [35]. L'absence d'hyperplasie des cellules cryptiques n'est pas due à la moindre capacité des cryptes à répondre, car un traitement par facteur de croissance épidermique (EGF) augmente la prolifération des cellules intestinales, mais sans améliorer l'état clinique [34]. La mort de cellules épithéliales compromet la digestion et l'absorption et est l'une des raisons de la diarrhée.

La coloration par le PAS (Periodic Acid Shiff) d'une coupe d'intestin grêle révèle une accumulation anormale de matériel PAS-positif dans le cytoplasme apical des cellules épithéliales. Dans l'atrophie microvillositaire congénitale, cette accumulation s'observe dans l'épithélium du collet des cryptes, tandis que, dans les cas «à début tardif», elle apparaît dans la partie basse de l'épithélium villositaire, et la coloration par le PAS de la bordure en brosse des cellules epithéliales sous jacentes est normale [12]. Dans une troisième variante, appelée atrophie microvillositaire «atypique», l'anomalie de la coloration par le PAS apparaît au fond des cryptes, alors qu' au niveau de l'épithélium superficiel, la coloration par le PAS de la bordure en brosse est quasiment normale [12]. L'anoma- 
lie de la coloration par le PAS suggère qu'une substance destinée à la bordure en brosse s'accumule dans la cellule au lieu d'être transportée vers la membrane apicale. Cette répartition coïncide avec l'observation en microscopie électronique (ME) d'une augmentation des «granules sécrétoires». Les deux anomalies représentent probablement le même phénomène [36]. Les granules sécrétoires contiennent donc un matériel qui devrait être normalement localisé à la bordure en brosse. La ME des «granules» a montré qu'ils ne sont pas pleins mais constituent un réseau vésiculaire [36]. L'appareil de Golgi est normal, indiquant que le blocage de l'exocytose se produit en aval. La coloration par le PAS en routine permet de faire un diagnostic rétrospectif [36].

La ME a mis en évidence l'autre signe majeur qu'est l'atrophie des microvillosités, la présence de vacuoles, ou d'inclusions, à revêtement microvillositaire dans le cytoplasme apical des cellules épithéliales superficielles (fig. 1), ce qui suggère que ces formations apparaissent plus tardivement que les granules sécrétoires dans le cycle de vie des cellules épithéliales. Elles peuvent prendre la forme d'inclusions petites ou volumineuses et, parfois, d'invaginations de la membrane apicale ou d'aspects «bulleux» avec des microvillosités d'un côté de l'inclusion (fig. 1b). Les inclusions microvillositaires subissent une digestion lysosomiale, et des fragments de microvillosité peuvent être présents dans des corps autolysosomiaux. Dans de nombreux cas, des microvillosités sont visibles sur les membranes latérales des cellules épithéliales.

Les inclusions microvillositaires et l'augmentation des granules sécrétoires ont été décrites dans l'intestin grêle comme dans le côlon [18], mais non dans l'estomac; elles ne l'ont pas été dans l'épithélium rénal ou le foie, mais sont effectivement présentes dans l'épithélium de la vésicule biliaire.

La bordure en brosse est anormale dans cette maladie, comme l'indique le terme d'atrophie microvillositaire. Il faut toutefois réaliser que, dans l'atrophie microvillositaire congénitale, les microvillosités sont d'aspect normal dans la région des cryptes et sont courtes ou absentes de façon variable sur la villosité. Dans les cas «à début tardif», l'aspect des microvillosités est normal dans la région villositaire basse. Dans l'atrophie microvillositaire «atypique», les microvillosités sont courtes ou absentes sur les cellules du collet des cryptes et réapparaissent sur les villosités. Ces variations de l'aspect des microvillosités sont parallèles à la répartition des granules sécrétoires : une fois les granules apparus, les microvillosités se raccourcissent, et si le nombre des granules diminue, les microvillosités réapparaissent [12].

Diarrhées intraitables dues à des anomalies congénitales des entérocytes

\section{Diagnostic}

Le diagnostic peut être facilement posé sur la coloration par le PAS et un examen en ME à transmission des biopsies de la muqueuse de l'intestin grêle proximal. L'approche la plus simple consiste à effectuer une coloration par le PAS de tissus bien orientés et fixés dans du formol, et de rechercher une accumulation de matériel PAS-positif dans le cytoplasme apical des entérocytes, habituellement dans la région du collet des cryptes ou du pied des villosités. Si cet aspect est présent, un examen en ME à transmission doit être effectué afin de déterminer s'il existe des inclusions microvillositaires. Celles-ci devraient être présentes dans des cellules épithéliales situées en surface, ce qui impose une orientation parfaite.

D'autres colorations ou marquages ont été proposés à titre d'outils diagnostiques: l'emploi de phosphatase alcaline [37], de l'antigène carcinoembryonnaire [38] ou de l'antigène CD10 [39], afin d'éviter la ME qui, bien qu'importante [40], est considérée comme techniquement exigeante. Ces techniques peuvent démontrer la présence d'inclusions microvillositaires, mais pas l'anomalie du PAS. La probabilité d'un diagnostic correct est plus élevée si l'on observe les deux anomalies, et il existe un risque d'erreur, si seules les techniques histochimiques sont employées, car elles peuvent aussi marquer les autophagosomes.

\section{Pathogénie}

La pathogénie de la maladie est inconnue. La présence d'inclusions microvillositaires pourrait témoigner d'une anomalie fondamentale de l'organisation du cytosquelette. Toutefois, l'observation de microvillosités normales dans des cellules aux stades précoces de leur développement dans les cas «congénitaux» et «à début tardif» et la réapparition des microvillosités dans les cas «atypiques» [12] indiquent que cette hypothèse est improbable. Les principales protéines du cytosquelette des microvillosités sont présentes dans le cytoplasme apical des cellules cryptiques et permettent la croissance et le renouvellement de la bordure en brosse [41]. Une perturbation de ce système peut donc aboutir à un assemblage prématuré de microvillosités dans la cellule (fig. 1a) ou à une croissance inappropriée de la membrane cellulaire latérale. Les inclusions microvillositaires pourraient aussi être dues à un enfouissement de la membrane apicale (fig. 1b) [4, 42]. La captation de matériel luminal dans les inclusions microvillositaires conforte cette hypothèse [42]. Ces éventualités ne s'excluent pas vraiment.

La présence des granules sécrétoires (fig. 2) suggère une anomalie de l'exocytose d'un matériel destiné à la bor- 
dure en brosse. Les voies constitutives directes et indirectes des enzymes de la bordure en brosse sont intactes [43] (bien que les concentrations enzymatiques soient diminuées en raison des lésions cellulaires [12]), ce qui implique une anomalie d'une voie transcellulaire non identifiée du trafic. Un des premiers articles sur le sujet a décrit un déficit en myosine de la bordure en brosse [44] suggérant qu'une anomalie des interactions entre actine et myosine pourrait être responsable de l'apport de substances membranaires à la bordure en brosse [45]. A l'occasion d'un cas isolé Ameen et Salas [46] ont aussi suggéré l'existence d'une anomalie du trafic membranaire apical.

La nature des granules sécrétoires est inconnue. Ils occupent normalement $2,5 \%$ du volume cellulaire apical, mais cette proportion s'élève à $40 \%$ dans l'atrophie microvillositaire [46]. Des données récentes démontrant la présence d'acide sialique acétylé et d'antigènes de groupe sanguin dans les granules sécrétoires indiquent qu'ils contiennent un matériel voisin du glycocalyx, qui ne subit pas d'exocytose [47]. Ces observations sont confortées par la nature floculente et fibrillaire de leur contenu (fig. 1a). Le glycocalyx est un gel polyanionique hydrophile qui recouvre la surface cellulaire et maintient sa charge ionique, exerce un effet protecteur contre les traumatismes physiques et régule les accès ioniques et macromoléculaires [48]. L'absence de glycocalyx aurait des conséquences très importantes pour la fonction cellulaire normale, et l'accumulation de débris cellulaires dans la cellule pourrait désorganiser les processus normaux. In utero, l'intestin contient du liquide amniotique dont l'osmolalité est voisine de celle du sérum, ce qui explique l'absence de gradient de pression osmotique trans-intestinal. Cette situation se modifie radicalement après la naissance, et l'absence de glycocalyx intestinal fonctionnel pourrait expliquer l'apparition rapide des troubles, et comment la plupart des cas d'atrophie microvillositaire pourraient résulter de l'anomalie d'exocytose du glycocalyx.

\section{Dysplasie épithéliale intestinale}

La dysplasie épithéliale intestinale a été décrite chez trois enfants par Reifen et coll. [50]. La diarrhée avait débuté au cours de la période néonatale. Un cas en avait été décrit par Davidson et coll. dans sa première série de diarrhée intraitable [4], soulignant l'hétérogénéité des pathologies responsables de ce syndrome. L'alimentation a entraîné une réduction spectaculaire du volume des sel- les, et un support nutritionnel a permis une croissance normale dans deux cas. L'entéropathie est caractérisée par l'existence de houpettes d'entérocytes tassés et un arrondissement de la membrane apicale qui semble se détacher de l'épithélium. Ces aspects non spécifiques avaient été décrits dans la maladie coeliaque, l'atrophie microvillositaire, l'entéropathie auto-immune et chez des sujets témoins, mais avec une fréquence beaucoup plus faible [50]. La formation de houpettes n'est pas observée dans l'épithélium des cryptes, il n'existe aucune anomalie ultrastructurale spécifique, l'infiltration cellulaire de la lamina propria n'est pas augmentée et l'entéropathie persiste malgré une amélioration possible sous support nutritionnel. Ces aspects permettent de classer cette affection dans la catégorie des anomalies entérocytaires congénitales et non dans celle des anomalies immunitaires. Des observations similaires chez 6 enfants ont été publiées [51] avec des données supplémentaires concernant des anomalies de la membrane basale (diminution de la laminine et augmentation du sulfate d'héparane), dont la nature primitive ou secondaire n'a pu, cependant, être établie. Patey et coll. [52] ont étudié les molécules d'adhésion et ont observé une augmentation de la présence de desmogléine, une répartition anormale de l' $\alpha 2 \beta 1$ intégrine le long de l'axe crypte-villosité et un accroissement de la présence de desmosomes. Ils en ont déduit qu'il existait des anomalies des interactions intercellulaires et entre la cellule et la matrice et qu'elles jouaient un rôle dans la pathogénie de la maladie.

Cette affection est généralement considérée comme autosomique récessive. La consanguinité parentale et l'atteinte de membres d'une même fratrie sont fréquentes [15]. Bien que Reifen et coll. [50] n'aient pas constaté d'anomalie du côlon chez leurs cas, des publications ultérieures ont montré que le côlon pouvait être atteint avec des anomalies des cryptes (ramifications et pseudokystes) [52]. Le diagnostic n'est pas nécessairement simple, car la prévalence des houpettes semble varier avec le temps, et des biopsies répétées sont nécessaires pour établir le diagnostic et exclure définitivement une atrophie microvillositaire [15]. Une inflammation de la lamina propria peut exister dans certains cas, probablement secondaire, et une augmentation des apports énergétiques peut être autorisée [53]. L'affection pourrait disparaître avec le temps dans certains cas. Au bout de 13 à 15 ans de traitement, deux adultes ne nécessitent plus maintenant de nutrition parentérale [53], et une publication a fait état d'une grossesse menée à bon terme chez une femme atteinte de dysplasie épithéliale intestinale [54]. 
Des analyses de génétique moléculaire permettront de mieux comprendre cette affection. Une association entre une dysplasie épithéliale intestinale et des malformations a été décrite [55], sans que l'on puisse s'avoir s'il s'agit d'une coïncidence ou d'une liaison génétique.

\section{Déficit en intégrine}

Lachaux et coll. [56] ont récemment décrit le cas d'un nourrisson présentant une atrésie du pylore et une diarrhée intraitable. La microscopie optique a révélé une desquamation étendue et sévère des cellules épithéliales de l'estomac au rectum, seules des cellules épithéliales des cryptes demeurant in situ. La ME de l'intestin a montré des desmosomes normaux alors que la lamina propria était décollée de la membrane basale des entérocytes. Ces troubles paraissent liés à un déficit congénital en $\alpha 6 \beta 4$ intégrine, mais portant sur une isoforme intestinale, car l'expression cutanée de cette substance s'est avérée normale. Cette intégrine est en cause dans l'épidermolyse bulleuse, où survient un décollement épidermique macroscopique. Il est probable qu'il existe une mutation de l'isoforme $\alpha 6 \beta 4$ intestinale ou un déficit d'une intégrine intestinale apparentée réagissant de façon croisée en immunohistochimie.

\section{Diarrhée syndromique}

Un petit nombre de patients ont été décrits comme présentant une diarrhée intraitable syndromique [57, 58]. Des cas similaires avaient été précédemment rapportés sous le nom de syndrome «tricho-hépato-entérique» [5961]. Dans ce syndrome, les enfants atteints présentent un retard de croissance intra-utérin et une diarrhée intraitable débutant au cours du premier mois de la vie, une hépatopathie, une dysmorphie faciale, un retard mental et une pilosité anormale. Les anomalies morphologiques de l'intestin grêle sont variables, allant d'une atrophie villositaire sévère à un raccourcissement modéré des villosités. Une colite a été également décrite [57]. Une consanguinité parentale est fréquente [58] et l'existence du syndrome dans une même fratrie a été décrite [59,61], ce qui évoque une affection autosomique récessive. Des réponses médiocres aux vaccinations et une hypogammaglobulinémie évocatrices de déficit immunitaire ont été observées dans certains cas.

Le pronostic paraît variable; certains patients ont succombé à une infection ou à une hépatopathie, d'autres nécessitent une nutrition parentérale totale ou partielle, et un patient s'alimente par voie orale [58]. Certains auteurs ont suggéré que la tolérance à la nutrition entérale était liée à l'état de l'intestin grêle [58]. La relation entre une diarrhée syndromique intraitable et le syndrome «tricho-hépato-entérique» doit être mieux étudiée. Le tableau clinique suggère une mutation de plusieurs gènes, hérités ensemble par déséquilibre de liaison, ou une anomalie de contrôle génétique, affectant par exemple un gène organisant la morphogenèse; les gènes de ce type impliqués dans le développement de la pilosité sont relativement peu nombreux, ce qui permet de mieux cibler la recherche des mutations candidates [15].

\section{Anomalies congénitales de la glycosylation et entéropathie exsudative}

Une diarrhée intraitable a été associée à des anomalies congénitales de la glycosylation, et une publication a décrit un cas de diarrhée sévère initiale chez un nourrisson de deux semaines et ayant persisté sous nutrition parentérale avec une hypoalbuminémie très marquée [62]. Des troubles neurologiques sont apparus, un traitement par mannose a été sans effet et le patient est décédé d'une insuffisance hépatique à l'âge de deux ans. Le trouble fondamental de la glycosylation, au-delà d'une anomalie de type 1, n'a pu être identifié.

Trois cas similaires de diarrhée intraitable et entéropathie exsudative sévère ont été publiés [63]. Dans ces cas, l'aspect histologique de la muqueuse de l'intestin grêle était normal, mais une absence de sulfate d'héparane a été mise en évidence sur les membranes latérales des cellules épithéliales. Le lien entre sulfate d'héparane, entéropathie exsudative et inflammation a été récemment établi de façon expérimentale [64]. Tous ces cas ont nécessité une nutrition parentérale, mais la diarrhée a persisté. Aucune autre manifestation du syndrome d'anomalie de la glycosylation n'a été décrite chez ces patients, bien qu'un cas de trouble congénital de type 1c de la glycosylation avec hypoalbuminémie sévère et absence de sulfate d'héparane dans l'épithélium intestinal ait été décrit [65]; les patients atteints d'anomalie de la glycosylation de type $1 \mathrm{~b}$ présentent une diarrhée sévère et une entéropathie exsudative [66]. Des similitudes existent donc entre ces diverses affections. 


\section{Autres causes et résumé}

Malgré les études approfondies des cas de diarrhée intraitable, un grand nombre d'entre eux ne sont pas caractérisés. Il faut espérer que la découverte des bases génétiques des maladies déjà reconnues permettra de mieux connaître les aspects de ces affections jusqu'à présent idiopathiques. L'implication des équipes de gastroenté- rologie pédiatrique soignant les enfants atteints de troubles du développement des entérocytes a certainement été à l'origine d'une meilleure prise en charge et de diagnostics plus précis, ce qui a permis des recherches ciblées et productives. Ces entéropathies persistent cependant souvent en dépit des interventions médicales, sauf si une transplantation intestinale est réalisée. La guérison de ces affections sévères attend la thérapie génique.

\section{Références}

- Avery GB, Villavicencio O, Lilly JR, Randolph JG: Intractable diarrhoea in early infancy. Pediatrics 1968;41:712-722.

-2 Larcher VF, Shepherd R, Francis DEM, Harries JT: Protracted diarrhoea in infancy. Analysis of 82 cases with particular reference to diagnosis and management. Arch Dis Child 1977;52:597-605.

-3 Hyman CJ, Reiter J, Rodnan J, Drash AL: Parenteral and oral alimentation in the treatment of the nonspecific protracted diarrheal syndrome of infancy. J Pediatr 1971;78:17-29.

-4 Davidson GP, Cutz E, Hamilton JR, Gall DG: Familial enteropathy: a syndrome of protracted diarrhea from birth, failure to thrive, and hypoplastic villous atrophy. Gastroenterology 1978;75:783-790.

-5 Candy DCA, Larcher VF, Cameron DJS, et al: Lethal familial protracted diarrhoea. Arch Dis Child 1981;56:15-23.

6 Cuenod B, Brousse N, Goulet O, et al: Classification of intractable diarrhea in infancy using clinical and immunohistological criteria. Gastroenterology 1990;99:1037-1043.

-7 Goulet OJ, Brousse N, Canioni D, WalkerSmith JA, Schmitz J, Phillips AD: Syndrome of intractable diarrhoea with persistent villous atrophy in early childhood: a clinicopathological survey of 47 cases. J Pediatr Gastroenterol Nutr 1998;26:151-161.

-8 Goulet O, Ruemmele F, Lacaille F, Colomb V: Irreversible intestinal failure. J Pediatr Gastroenterol Nutr 2004;38:250-269.

-9 Sanderson IR, Risdon RA, Walker-Smith JA: Intractable ulcerating enterocolitis of infancy. Arch Dis Child 1991;66:295-299.

10 Thapar N, Shah N, Ramsay AD, Lindley KJ, Milla PJ: Long-term outcome of intractable ulcerating enterocolitis of infancy. J Pediatr Gastroenterol Nutr 2005;40:582-588.

11 Cutz E, Rhoads JM, Drumm B, Sherman PM, Durie PR, Forstner GG: Microvillus inclusion disease: an inherited defect of brush-border assembly and differentiation. N Engl J Med 1989;320:646-651.

12 Phillips AD, Schmitz J: Familial microvillous atrophy: a clinicopathological survey of 23 cases. J Pediatr Gastroenterol Nutr 1992;14: 380-396.
13 Cutz E, Sherman PM, Davidson GP: Enteropathies associated with protracted diarrhea of infancy: clinicopathological features, cellular and molecular mechanisms. Pediatr Pathol Lab Med 1997;17:335-368.

14 Sherman PM, Mitchell DJ, Cutz E: Neonatal enteropathies: defining the causes of protracted diarrhea of infancy. J Pediatr Gastroenterol Nutr 2004;38:16-26.

15 Goulet O, Phillips AD: Congenital enteropathy involving intestinal mucosa development; in Walker WA, Goulet O, Kleinman RE, Sanderson IR, Sherman P, Shneider B (eds): Paediatric Gastrointestinal Disease. Burlington, Decker, 2004, pp 922-931.

16 Schmitz J, Ginies JL, Arnaud-Battandier F, et al: Congenital microvillous atrophy: a rare cause of neonatal intractable diarrhoea. Pediatr Res 1982;16:1014.

17 Goutet JM, Boccon-Gibod L, Chatelet F, Ploussard JP, Navarro J, Polonovski Cl: Familial protracted diarrhoea with hypoplastic villous atrophy: report of two cases. Pediatr Res 1982;16:1045.

18 Phillips AD, Jenkins P, Raafat F, WalkerSmith JA: Congenital microvillous atrophy: specific diagnostic features. Arch Dis Child 1985;60:135-140.

19 Beck NS, Chang YS, Kang IS, Park WS, Lee HJ, Suh YL: Microvillus inclusion disease in two Korean infants. J Korean Med Sci 1997 12:452-456.

20 Kaneko K, Shimizu T, Fujiwara S, Igarashi J, Ohtomo Y, Yamashiro Y: Microvillous inclusion disease in Japan. J Pediatr 1999;135: 400.

21 Pohl JF, Shub MD, Trevelline EE, et al: A cluster of microvillous inclusion disease in the $\mathrm{Na}-$ vajo population. J Pediatr 1999;134:103106.

22 Kennea N, Norbury R, Anderson G, Tekay A: Congenital microvillous inclusion disease presenting as antenatal bowel obstruction. Ultrasound Obstet Gynecol 2001;17:172-174.

23 Makela S, Kere J, Holmberg C, Hoglund P: SLC26A3 mutations in congenital chloride diarrhea. Hum Mutat 2002;20:425-438.
24 Keller KM, Wirth S, Baumann W, Sule D, Booth IW: Defective jejunal brush border membrane sodium/proton exchange in association with lethal familial protracted diarrhoea. Gut 1990;31:1156-1158.

-25 Croft NM, Howatson AG, Ling SC, Nairn L, Evans TJ, Weaver LT: Microvillous inclusion disease: an evolving condition. J Pediatr Gastroenterol Nutr 2000;31:185-189.

26 Ruemmele FM, Jan D, Lacaille F, et al: New perspectives for children with microvillous inclusion disease: early small bowel transplantation. Transplantation 2004;77:1024-1028.

27 Randak C, Langnas AN, Kaufman SS, et al: Pretransplant management and small bowelliver transplantation in an infant with microvillus inclusion disease. J Pediatr Gastroenterol Nutr 1998:27:333-337.

- 28 Nathavitharana KA, Green NJ, Raafat F, Booth IW: Siblings with microvillous inclusion disease. Arch Dis Child 1994;71:71-73.

-29 Assmann B, Hoffmann GF, Wagner L, et al: Dihydropyrimidinase deficiency and congenital microvillous atrophy: coincidence or genetic relation? J Inherit Metab Dis 1997;20:681688 .

30 Heinz-Erian P, Schmidt H, Le Merrer M, Phillips AD, Kiess W, Hadorn HB: Congenital microvillus atrophy in a girl with autosomal dominant hypochondroplasia. J Pediatr Gastroenterol Nutr 1999;28:203-205

- 31 Raafat F, Green NJ, Nathavitharana KA, Booth IW: Intestinal microvillous dystrophy: a variant of microvillous inclusion disease or a new entity? Hum Pathol 1994;25:1243-1248.

- 32 Mierau GW, Wills EJ, Wyatt-Ashmead J, Hoffenberg EJ, Cutz E: Microvillous inclusion disease: report of a case with atypical features. Ultrastruct Pathol 2001;25:517-521.

-33 Weeks DA, Zuppan CW, Malott RL, Mierau GW: Microvillous inclusion disease with abundant vermiform, electron-lucent vesicles. U1trastruct Pathol 2003;27:337-340.

34 Walker-Smith JA, Phillips AD, Walford N, et al: Intravenous epidermal growth factor/urogastrone increases small intestinal cell proliferation in congenital microvillous atrophy. Lancet 1985;ii:1239-1240. 
>35 Groisman GM, Sabo E, Meir A, Polak-Charcon S: Enterocyte apoptosis and proliferation are increased in microvillous inclusion disease (familial microvillous atrophy). Hum Pathol 2000;31:1404-1410.

-36 Phillips AD, Szafranski M, Man LY, Wall WJ: Periodic acid-Schiff staining abnormality in microvillous atrophy: photometric and ultrastructural studies. J Pediatr Gastroenterol Nutr 2000;30:34-42.

-37 Lake BD: Microvillus inclusion disease: specific diagnostic features shown by alkaline phosphatase histochemistry. J Clin Pathol 1988;41:880-882.

-38 Groisman GM, Ben-Izhak O, Schwersenz A, Berant M, Fyffe B: The value of polyclonal carcinoembryonic antigen immunostaining in the diagnosis of microvillus inclusion disease. Hum Pathol 1993;24:1232-1237.

-39 Groisman GM, Amar M, Livne E: CD10: a valuable tool for the light microscopic diagnosis of microvillous inclusion disease (familial microvillous atrophy). Am J Surg Pathol 2002; 26:902-907.

-40 Bell SW, Kerner JA, Sibley RK: Microvillous inclusion disease. The importance of electron microscopy for diagnosis. Am J Surg Pathol 1991;15:1157-1164.

-41 Fath KR, Obenauf SD, Burgess DR: Cytoskeletal protein and mRNA accumulation during brush border formation in adult chicken enterocytes. Development 1990;109:449-459.

-42 Reinshagen K, Naim HY, Zimmer KP: Autophagocytosis of the apical membrane in microvillus inclusion disease. Gut 2002;51:514521.

43 Phillips AD, Fransen JAM, Hauri HP, Sterchi E: The constitutive exocytotic pathway in microvillous atrophy. J Pediatr Gastroenterol Nutr 1993; 17:239-246.

44 Carruthers L, Phillips AD, Dourmashkin R, Walker-Smith JA: Biochemical abnormality in brush border membrane protein of a patient with congenital microvillous atrophy. J Pediatr Gastroenterol Nutr 1985;4:902-907.

-45 Fath KR, Trimbur GM, Burgess DR: Molecular motors are differentially distributed on Golgi membranes from polarized epithelial cells. J Cell Biol 1994;126:661-675.
46 Ameen NA, Salas PJ: Microvillus inclusion disease: a genetic defect affecting apical membrane protein traffic in intestinal epithelium. Traffic 2000; 1:76-83.

47 Phillips AD, Brown A, Hicks S, et al: Acetylated sialic acid residues and blood group antigens localise within the epithelium in microvillous atrophy indicating internal accumulation of the glycocalyx. Gut 2004;53:1764-1771.

48 Gupta BL: The relationship of mucoid substances and ion and water transport, with new data on intestinal goblet cells and a model for gastric secretion. Symp Soc Exp Biol 1989;43: 81-110.

49 Gillibrand PN: Changes in the electrolytes, urea and osmolality of the amniotic fluid with advancing pregnancy. J Obstet Gynaecol $\mathrm{Br}$ Commonw 1969;76:898-905.

50 Reifen RM, Cutz E, Griffiths AM, Ngan BY, Sherman PM: Tufting enteropathy: a newly recognized clinicopathological entity associated with refractory diarrhea in infants. J Pediatr Gastroenterol Nutr 1994;18:379-385.

51 Goulet O, Kedinger M, Brousse N, et al: Intractable diarrhea of infancy with epithelial and basement membrane abnormalities. J Pediatr 1995; 127:212-219.

52 Patey N, Scoazec JY, Cuenod-Jabri B, et al: Distribution of cell adhesion molecules in infants with intestinal epithelial dysplasia (tufting enteropathy). Gastroenterology 1997;113: 833-843.

53 Schaeppi M, Shah NF, Long S, et al: Tufting enteropathy also affects the colon. J Pediatr Gastroenterol Nutr 1999;28:569.

54 Cameron DJ, Barnes GL: Successful pregnancy outcome in tufting enteropathy. J Pediatr Gastroenterol Nutr 2003;36:158.

55 Abely M, Hankard GF, Hugot JP, Cezard JP, Peuchmaur M, Navarro J: Intractable infant diarrhea with epithelial dysplasia associated with polymalformation. J Pediatr Gastroenterol Nutr 1998;27:348-352.

56 Lachaux A, Bouvier R, Loras-Duclaux I, Chappuis JP, Meneguzzi G, Ortonne JP: Isolated deficient alpha6beta4 integrin expression in the gut associated with intractable diarrhea. J Pediatr Gastroenterol Nutr 1999;29:395401.
57 Girault D, Goulet O, Le Deist F, et al: Intractable infant diarrhea associated with phenotypic abnormalities and immunodeficiency. $\mathrm{J}$ Pediatr 1994; 125:36-42.

58 Martinez-Vinson C, Goulet O, Berrebi D, et al: Syndromatic diarrhea in children. Report of 8 cases. J Pediatr Gastroenterol Nutr 2005;40: 651.

59 Verloes A, Lombet J, Lambert Y, et al: Trichohepato-enteric syndrome: further delineation of a distinct syndrome with neonatal hemochromatosis phenotype, intractable diarrhea, and hair anomalies. Am J Med Genet 1997;68: 391-395.

60 Landers MC, Schroeder TM: Intractable diarrhea of infancy with facial dysmorphism, trichorrhexis nodosa, and cirrhosis. Pediatr Dermatol 2003;20:432-435.

61 Stankler L, Lloyd D, Pollitt RJ, Gray ES, Thom H, Russell G: Unexplained diarrhoea and failure to thrive in 2 siblings with unusual facies and abnormal scalp hair shafts: a new syndrome. Arch Dis Child 1982;57:212-216.

-62 Mention K, Michaud L, Dobbelaere D, Guimber D, Gottrand F, Turck D: Neonatal severe intractable diarrhoea as the presenting manifestation of an unclassified congenital disorder of glycosylation (CDG-x). Arch Dis Child Fetal Neonatal Ed 2001;85:F217-F219.

63 Murch SH, Winyard PJ, Koletzko S, et al: Congenital enterocyte heparan sulphate deficiency with massive albumin loss, secretory diarrhoea, and malnutrition. Lancet 1996;347: 1299-1301.

64 Bode L, Eklund EA, Murch S, Freeze HH: Heparan sulfate depletion amplifies TNF-alpha-induced protein leakage in an in vitro model of protein-losing enteropathy. Am J Physiol Gastrointest Liver Physiol 2005;288: G1015-G1023.

65 Westphal V, Murch S, Kim S, et al: Reduced heparan sulfate accumulation in enterocytes contributes to protein-losing enteropathy in a congenital disorder of glycosylation. Am J Pathol 2000;157:1917-1925.

66 Niehues R, Hasilik M, Alton G, et al: Carbohydrate-deficient glycoprotein syndrome type Ib. Phosphomannose isomerase deficiency and mannose therapy. J Clin Invest 1998;101: 1414-1420. 